\title{
LATE CRETACEOUS OSTRACODS FROM THE CENTRAL AREA OF THE POTIGUAR BASIN, NORTHEASTERN BRAZIL
}

\author{
ENELISE KATIA PIOVESAN (D) \\ Laboratório de Micropaleontologia Aplicada (LMA), Programa de Pós-Graduação em Geociências, \\ Universidade Federal de Pernambuco, Av. da Arquitetura, s/n, 50740-550, Recife, PE, Brazil. \\ katiapiovesan@gmail.com \\ GERSON FAUTH (D) \\ Instituto Tecnológico de Micropaleontologia (itt Fossil), Universidade do Vale do Rio dos Sinos, \\ Av. Unisinos, 950, 93022-750, São Leopoldo, RS, Brazil. \\ gersonf@unisinos.br \\ CRISTIANINI TRESCASTRO BERGUE (D) \\ Departamento Interdisciplinar, Centro de Estudos Costeiros, Limnológicos e Marinhos - CECLIMAR, \\ Universidade Federal do Rio Grande do Sul, Av.Tramandaí, 976, 95625-000, Imbé, RS, Brazil. \\ ctbergue@gmail.com
}

\begin{abstract}
The Jandaíra Formation samples collected in the central region of the Potiguar Basin show a diverse Late Cretaceous ostracod fauna. The genera Cytherella, Bairdoppilata, Triebelina, Paracypris, Fossocytheridea, Ovocytheridea, Perissocytheridea, Protocosta, Soudanella, and Leguminocythereis indicate a marginal marine environment during the Santonian-Campanian. The analyzed material demonstrates that ostracods are significant indicators of changes in the depositional environment and paleoecological markers in marginal marine environments.
\end{abstract}

Key words: taxonomy, Upper Cretaceous, paleoecology, neritic assemblages.

RESUMO - As amostras provenientes da Formação Jandaíra, coletadas na região central da Bacia Potiguar, evidenciam uma diversificada fauna de ostracodes do Neocretáceo. Os gêneros Cytherella, Bairdoppilata, Triebelina, Paracypris, Fossocytheridea, Ovocytheridea, Perissocytheridea, Protocosta, Soudanella e Leguminocythereis indicam um ambiente marinho marginal no intervalo Santoniano-Campaniano. O material analisado demonstra que os ostracodes são indicadores significativos de modificações no ambiente deposicional e marcadores paleoecológicos em ambientes marinhos marginais.

Palavras-chave: taxonomia, Cretáceo Superior, paleoecologia, assembleias neriticas.

\section{INTRODUCTION}

In the last decades research on Cretaceous ostracods of Brazilian basins have received considerable attention, especially those in marginal marine environments. Their biostratigraphical potential in the South Atlantic was demonstrated recently in the revision by Fauth et al. (2018). The Late Cretaceous ostracods from the Potiguar Basin were studied by Viviers (1995, 2000), Delicio et al. (2000), Piovesan et al. (2014a,b; 2015a,b), and Santos Filho et al. (2015), who improved significantly the knowledge on the evolution of this basin, and reinforced the correlation between Brazilian and North African ostracod faunas in the Upper Cretaceous.
Piovesan et al. (2015a) demonstrated the existence of four major palaeoenvironmental phases in the TuronianCampanian interval of the Potiguar Basin, as follows: (i) mixohaline/marine 1, (ii) marine 1, (iii) marine 2, and (iv) mixohaline/marine 2. Assemblages in each of those phases differ on taxonomic composition and abundance, demonstrating their potential for the characterization of marine and marginal marine environments and sequence stratigraphic interpretation.

This paper is based upon a study of a new locality of the Jandaíra Formation, as part of an effort initiated by Piovesan $e t$ al. $(2014 \mathrm{a}, \mathrm{b})$ whose objective is to supply new paleoecological and bioestratigraphical data to the Potiguar Basin based on brackish and marine ostracods. 


\section{GEOLOGICAL SETTING}

The Potiguar Basin (Figure 1) is located at the intersection of the Equatorial Margin with the East Continental Margin, covering an area of approximately $48,000 \mathrm{~km}^{2}$. Geologically, it is limited on the south, east and west by the crystalline basement, extending northwards to the $2,000 \mathrm{~m}$ isobath. This basin is distributed mostly in the Rio Grande do Norte and Ceará states (Mohriak, 2003; Pessoa Neto et al., 2007).

According to Pessoa Neto et al. (2007), the Potiguar Basin is comprised of three supersequences, as follows: (i) rift (Berriasian-Lower Aptian), represented by fluvialdeltaic and lacustrine deposits from the Pendência and Pescada formations; (ii) post-rift, deposited during the late Aptian-early Albian and characterized by fluvial-deltaic deposits, with the first records of the marine transgression (Alagamar Formation), and (iii) drift, including all the marine sedimentation from the early Albian onwards. The studied material was deposited during the last sequence, which represents the maximum expansion of the carbonate domain in the Potiguar Basin.

The Jandaíra Formation was deposited during a marine ingression and is composed of calcilutites and calcarenites deposited in marginal marine environments on a shallow platform (Monteiro \& Faria, 1988; Araripe \& Feijó, 1994; Piovesan et al., 2015a) during the Turonian-Campanian interval.

\section{MATERIAL AND METHODS}

The samples herein studied are from an outcrop (UTM 0673396/9411152) located in Rio Grande do Norte State, Brazil. The ages are based on Viviers et al. (2000) and Piovesan et al. (2014b). The outcrop was sampled at four different levels, named A, B, C and D from the base to the top (Figure 2). Lithologically, the rock samples are mostly composed of calcarenites, and occasionally interstratified layers of oysters occur. A total of $100 \mathrm{~g}$ of sediment from each sample was disaggregated with hydrogen peroxide $\left(\mathrm{H}_{2} \mathrm{O}_{2}\right)$, washing through sieves with mesh 250,180 and 63 micrometers and oven-dried at $60^{\circ} \mathrm{C}$. All ostracod specimens from the three fractions were picked out and put on micropaleontological slides for taxonomic identification. Specimens representative of each taxa were selected for SEM (scanning electron microscopy) at Laboratório de Dispositivos e Nanoestruturas of Universidade Federal de Pernambuco (LDN-UFPE) and itt Fossil, UNISINOS.

The suprageneric taxonomy herein adopted follows Horne (2005). In the systematic descriptions, the following abbreviations/conventions are used: $\mathbf{L}$, length; $\mathbf{H}$, height; $\mathbf{W}$, width; $\mathbf{C}$, carapace; $\mathbf{R V}$, right view; $\mathbf{L V}$, left view; DV, dorsal view; f, female; $\mathbf{m}$, male. Type and figured specimens are deposited in the collections of Laboratório de Micropaleontologia Aplicada (LMA) - Universidade Federal de Pernambuco (UFPE), Brazil, under the curatorial numbers LMA-00001 to LMA 00019.
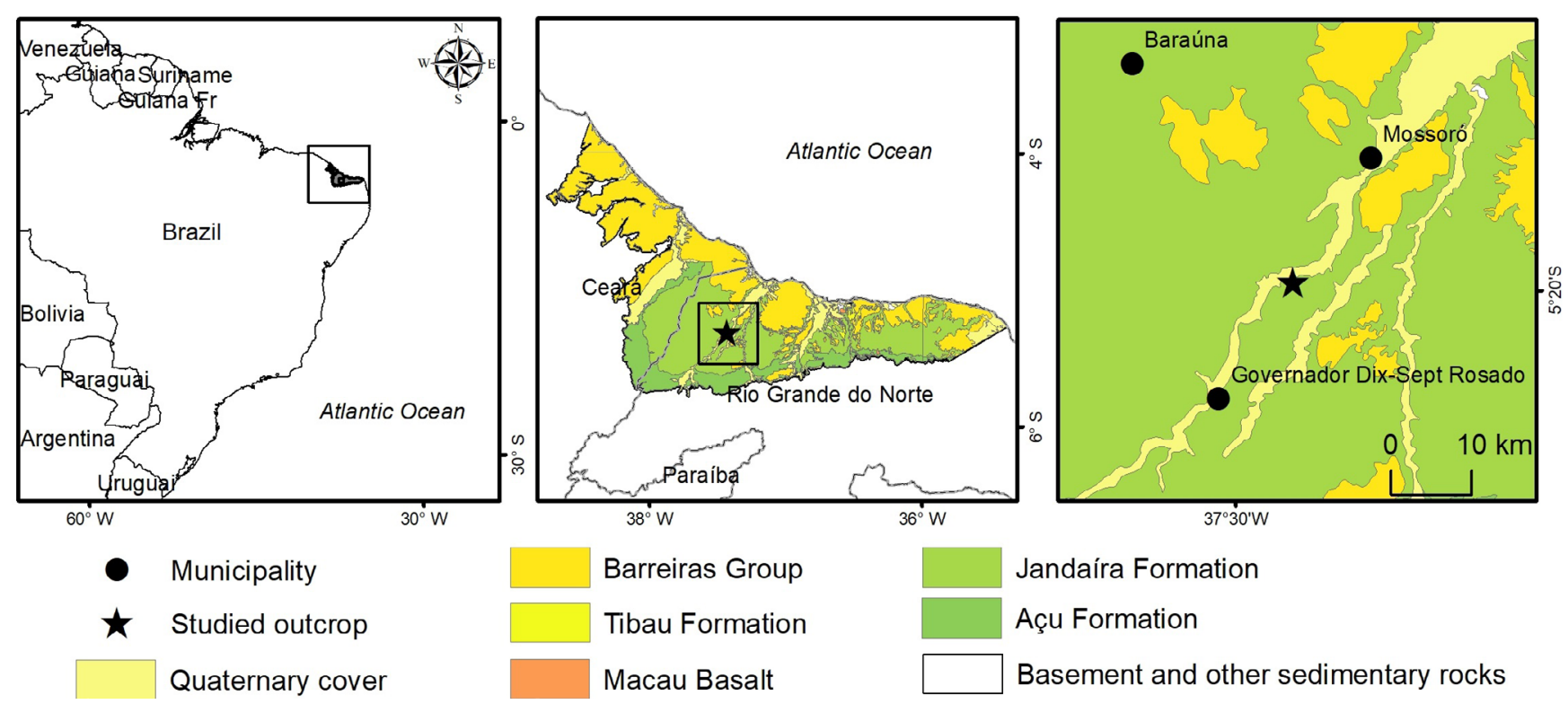

Figure 1. Map of the Potiguar Basin indicating the study area, elaborated in QGIS software (metadata from Geological Survey of Brazil - CPRM). 


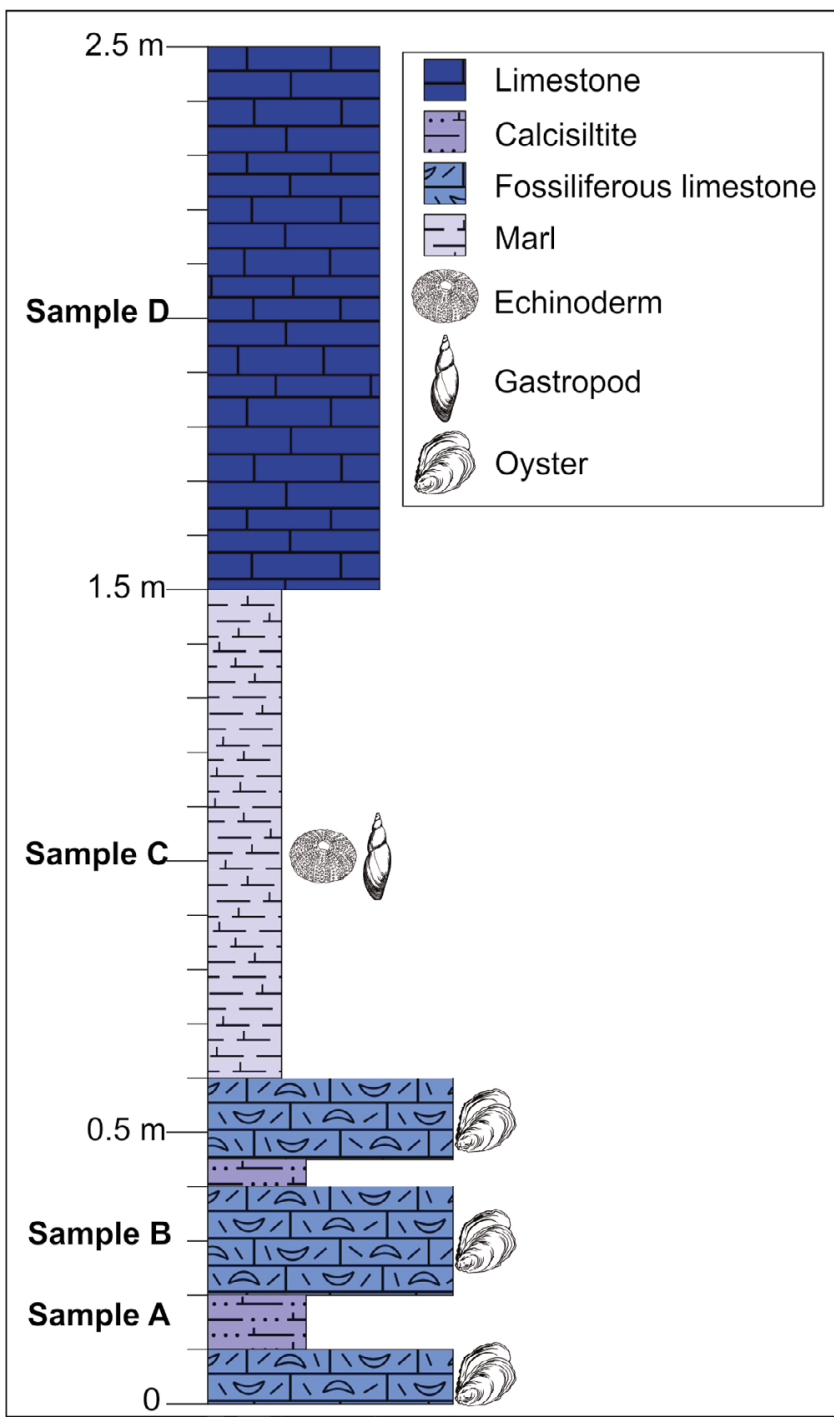

Figure 2. Lithologic profile of the studied outcrop with indication of the position of the sampling points and the macrofossils observed.

\section{SYSTEMATIC PALEONTOLOGY}

\author{
Class OSTRACODA Latreille, 1802 \\ Subclass PODOCOPA Sars, 1866 \\ Order PLATYCOPIDA Sars, 1866 \\ Suborder PLATYCOPINA Sars, 1866 \\ Superfamily CYTHERELLOIDEA Sars, 1866 \\ Family CYTHERELLIDAE Sars, 1866
}

Cytherella Jones, 1849

Cytherella gambiensis Apostolescu, 1963

(Figure 3A)

1963 Cytherella gambiensis Apostolescu, p. 1680, pl. 1, figs. $1-3$.

non 1981 Cytherella cf. gambiensis Apostolescu-Bismuth et al., p. 221-222, pl. 6, figs. 1-2.
1985 Cytherella cf. gambiensis Apostolescu - Vivière, p. 137-138, pl. 1, fig. 7.

2000 Cytherella gambiensis Apostolescu - Delicio et al., p. 331-332, figs. 8.3-8.4.

2000 Cytherella aff. C. gambiensis Viviers et al., p. 415, figs. 8, 5-6 and 9-11.

2014b Cytherella gambiensis Apostolescu - Piovesan et al., p. 318 , pl. 1, figs. A-D.

Material. 46 specimens (samples A and C).

Illustrated specimen. LMA-00001, C, LV, Figure 3A; 1 : $0.89 \mathrm{~mm}, \mathrm{~h}: 0.61 \mathrm{~mm}$.

Age. Santonian-Campanian.

Stratigraphic and geographic distribution. Turonian of Algeria (Vivière, 1985), Senonian of Gambia (Apostolescu, 1963), Upper Cretaceous of the Potiguar Basin (Delicio et al., 2000), Coniacian-Campanian (Viviers et al., 2000) and Santonian-Campanian of the Potiguar Basin (Piovesan et al., 2014b; Santos Filho et al., 2015; in this work).

Remarks. Some Cytherella species seem to characterize the Late Cretaceous epoch in Brazilian marginal basins. $C$. gambiensis seems to be an important marker for SantonianCampanian, and Cytherella ovoidea Alexander, 1929 is typical of Maastrichtian deposits in the Paraíba Basin (Fauth et al., 2005; Barros et al., 2018). This demonstrates the biostratigraphic potential of this widespread genus.

\section{Cytherella mediatlasica Andreu, 1996}

(Figure 3B)

1987 Cytherella sp. Okosun, p. 25, pl. 13, figs. 5-6.

1992 Cytherella sp. Okosun, p. 328, pl. 2, fig. 20.

1996 Cytherella mediatlasica Andreu, p. 484-485, 488, pl. 1 , figs. 1-10.

2000 Cytherella sp. P6 Viviers, Koutsoukos, Silva-Telles \& Bengtson, p. 415, fig. 8, 14-15.

2014a Cytherella mediatlasica Andreu - Piovesan et al., p. 214, pl. 1, figs. A-D.

2014b Cytherella mediatlasica Andreu - Piovesan et al., p. 318, pl. 1, figs. E-I.

Material. one specimen (sample C).

Illustrated specimen. LMA-00002, C, LV, Figure 3B; 1: $0.63 \mathrm{~mm}, \mathrm{~h}: 0.34 \mathrm{~mm}$.

Age. Santonian-Campanian.

Stratigraphic and geographic distribution. TuronianSantonian of Nigeria (Okosun, 1987, 1992), Santonian of Morocco (Andreu, 1996), Turonian-Campanian of the Potiguar Basin (Viviers et al., 2000; Piovesan et al., 2014a,b; Santos Filho et al., 2015) and Santonian-Campanian in this work.

Remarks. Andreu (1996) was the first author to comment on the polymorphism of this species, a characteristic observed years later by Piovesan et al. (2014a,b; 2015a).

Order PODOCOPIDA Sars, 1866 Suborder BAIRDIOCOPINA Sars, 1887 Superfamily BAIRDIOIDEA Sars, 1887 
Family BAIRDIIDAE Sars, 1887

Bairdoppilata Coryell, Sample \& Jennings, 1935

Bairdoppilata sp. 1

(Figure 3C)

Material. 11 specimens (sample C).

Illustrated specimen. LMA-00003, C, RV, Figure 3C; 1: $0.92 \mathrm{~mm}$, h: $0.66 \mathrm{~mm}$.

Age. Santonian-Campanian.

Remarks. Bairdoppilata sp. 1 differs from other species of this genus registered in the Potiguar Basin mainly in having the anterior and posterior ends blunt.

Triebelina Bold, 1946

Triebelina anterotuberculata Piovesan, Cabral \& Colin, 2014

(Figure 3D)

2014b Triebelina anterotuberculata Piovesan, Cabral \& Colin, p. 322, pl. 2, figs. H-L.

Material. One specimen (sample C).

Illustrated specimen. LMA-00004, C, RV, Figure 3D; 1: $0.95 \mathrm{~mm}, \mathrm{~h}: 0.54 \mathrm{~mm}$.

Age. Santonian-Campanian.

Stratigraphic and geographic distribution. SantonianCampanian, Potiguar Basin (Piovesan et al., 2014b; in this work).

Remarks. Despite the poor preservation, this specimen is identified at species level by the outline and the anterior tubercle.

Suborder CYPRIDOCOPINA Baird, 1850

Superfamily CYPRIDOIDEA Baird, 1845

Family CANDONIDAE Kaufmann, 1900

Subfamily PARACYPRIDINAE Sars, 1923

Paracypris Sars, 1866

Paracypris sp. 1

(Figure 3E)

Material. Nine specimens (sample C).

Illustrated specimen. LMA-00005, C, Figure 3E; 1: $0.80 \mathrm{~mm}, \mathrm{~h}: 0.42 \mathrm{~mm}$.

Age. Santonian-Campanian.

Remarks. This species differs from others identified in the Potiguar Basin by the rounded posterior end. The material is scarce and poorly preserved and, for these reasons, cannot be described.

Suborder CYTHEROCOPINA Baird, 1850

Superfamily CYTHEROIDEA Baird, 1850
Family CYTHERIDEIDAE Sars, 1925

Cophinia Apostolescu, 1961

Cophinia ovalis Piovesan, Cabral \& Colin, 2014

(Figures 3F-G)

2000 Cophinia aff. C. apiformis Viviers, Koutsoukos, SilvaTelles \& Bengtson, p. 423, fig. 13, 1-4.

2014b Cophinia ovalis Piovesan, Cabral \& Colin, p. 326, pl. 3, figs. D-I.

Material. 167 specimens (samples A and C).

Illustrated specimens. LMA-00006, C, RV, Figure 3F; 1 : $0.89 \mathrm{~mm}$, h: $0.75 \mathrm{~mm}$; LMA-00007, C, DV, Figure 3G; 1 : $1.02 \mathrm{~mm}$, w: $0.45 \mathrm{~mm}$.

Age. Santonian-Campanian.

Stratigraphic and geographic distribution. SantonianCampanian, Potiguar Basin (Viviers et al., 2000; Piovesan et al., 2014b; in this work).

\section{Fossocytheridea Swain \& Brown, 1964}

Fossocytheridea potiguarensis Piovesan, Cabral \& Colin, 2014

(Figures 3H-I)

2000 Sarlatina sp. P5 Viviers, Koutsoukos, Silva-Telles \& Bengtson, p. 424, fig. 14, 5-8.

2014b Fossocytheridea potiguarensis Piovesan, Cabral \& Colin, p. 326, pl. 3, figs. J-P.

Material. Nine specimens (sample A).

Illustrated specimens. LMA-00008, right valve, Figure $3 \mathrm{H}$; m, 1: $1.12 \mathrm{~mm}$, h: $0.55 \mathrm{~mm}$; LMA-00009, C, RV, Figure 3I, f, 1: $1 \mathrm{~mm}$, h: $0.58 \mathrm{~mm}$.

Age. Santonian-Campanian.

Stratigraphic and geographic distribution. SantonianCampanian, Potiguar Basin (Viviers et al., 2000; Piovesan et al., 2014b; in this work).

Ovocytheridea Grékoff, 1951

Ovocytheridea anterocompressa Piovesan, Cabral \& Colin, 2014

(Figure 3J)

2014b Ovocytheridea anterocompressa Piovesan, Cabral \& Colin, p. 330, pl. 4, figs. I-M.

Material. 742 specimens (samples A, B and C).

Illustrated specimen. LMA-00010, C, RV, Figure 3J; $1: 0.76$ $\mathrm{mm}, \mathrm{h}: 0.46 \mathrm{~mm}$.

Age. Santonian-Campanian.

Stratigraphic and geographic distribution. SantonianCampanian, Potiguar Basin (Piovesan et al., 2014b; in this work). 


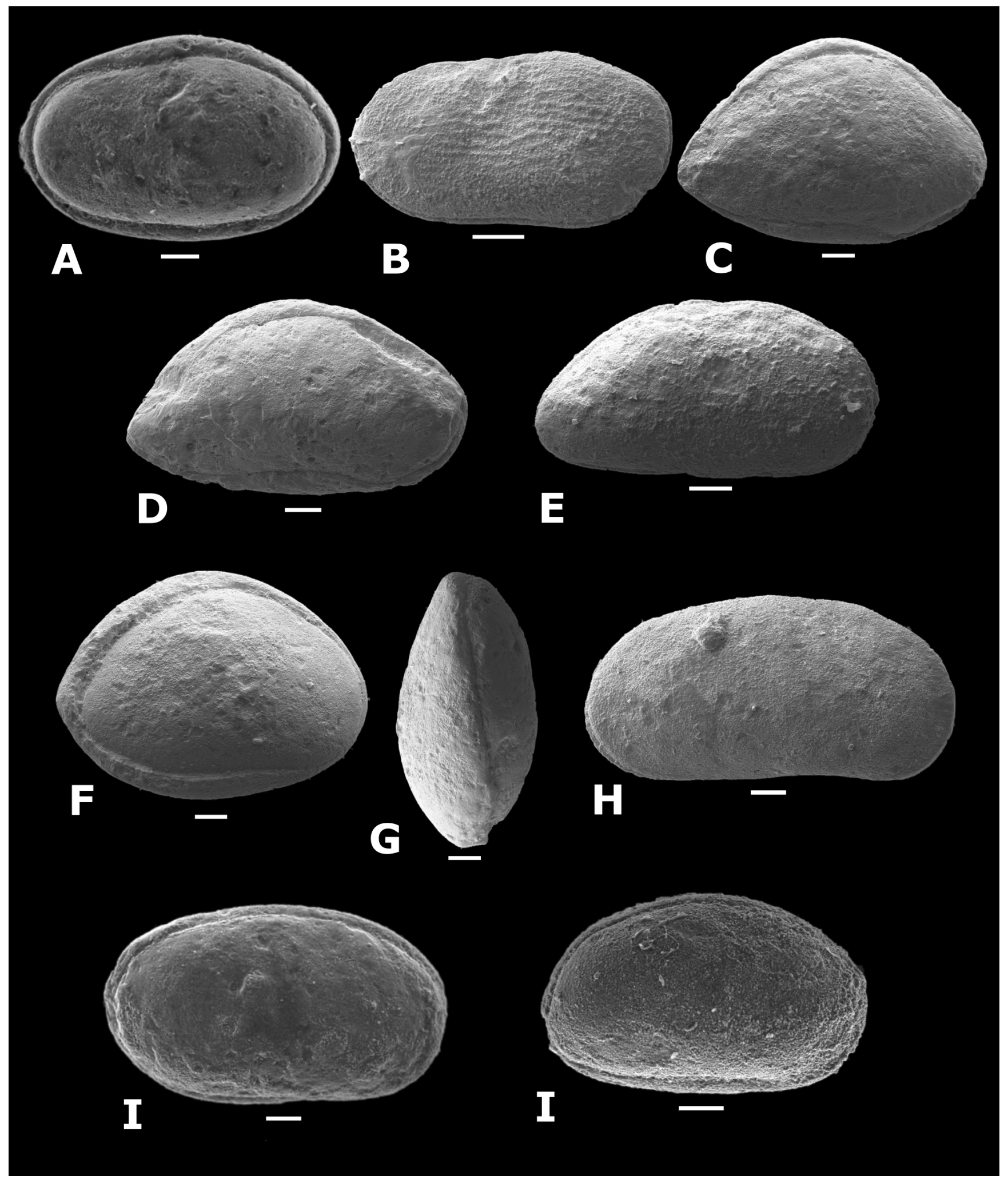

Figure 3. Ostracod species A, Cytherella gambiensis, C, LV (LMA-00001); B, Cytherella mediatlasica, C, LV (LMA-00002); C, Bairdoppilata sp. 1, C, RV (LMA-00003); D, Triebelina anterotuberculata, C, RV (LMA-00004); E, Paracypris sp. 1, C, RV (LMA-00005); F, Cophinia ovalis, C, RV (LMA-00006); G, Cophinia ovalis, C, DV (LMA-00007); H, Fossocytheridea potiguarensis, RV, m, RV (LMA-00008); I, Fossocytheridea potiguarensis, C, f, RV (LMA00009); J, Ovocytheridea anterocompressa, C, RV (LMA-00010). Scale bars $=100 \mu \mathrm{m}$. 
Ovocytheridea triangularis Piovesan, Cabral \& Colin, 2014 (Figures 4A-B)

2000 Ovocytheridea aff. O. producta Grékoff, 1962 - Viviers et al., p. 423, fig. 13, 21-22.

2014b Ovocytheridea triangularis Piovesan, Cabral \& Colin, p. 330 , pl. 4 , figs. N-R.

Material. 34 specimens (sample C).

Illustrated specimens. LMA-00011, C, RV, Figure 4A; 1: $0.77 \mathrm{~mm}$, h: $0.46 \mathrm{~mm}$; LMA-00012, C, DV, Figure 4B; 1: $0.78 \mathrm{~mm}$, w: $0.36 \mathrm{~mm}$.

Age. Santonian-Campanian.

Stratigraphic and geographic distribution. SantonianCampanian, Potiguar Basin (Viviers et al., 2000; Piovesan et al., 2014b; in this work).

Perissocytheridea Stephenson, 1938

Perissocytheridea jandairensis Piovesan, Cabral \& Colin, 2014

(Figure 4C)

2000 Semicytherura sp. P1 Viviers, Koutsoukos, Silva-Telles \& Bengtson, p. 424, fig. 14, 19-23.

2014b Perissocytheridea jandairensis Piovesan, Cabral \& Colin, p. 332, pl. 5, figs. E-I.

Material. Six specimens (samples A, B and C).

Illustrated specimen. LMA-00013, C, RV, Figure 4C; 1 : $0.47 \mathrm{~mm}$, h: $0.23 \mathrm{~mm}$.

Age. Santonian-Campanian.

Stratigraphic and geographic distribution. SantonianCampanian, Potiguar Basin (Viviers et al., 2000; Piovesan et al., 2014b; in this work). Family TRACHYLEBERIDIDAE Sylvester-Bradley,
1948

Protocosta Bertels, 1969

Protocosta babinoti Piovesan, Cabral \& Colin, 2014

(Figures 4D-E)

2000 Cythereis? sp. P8 Viviers, Koutsoukos, Silva-Telles \& Bengtson, p. 433, fig. 19, 3-4.

2014b Protocosta babinoti Piovesan, Cabral \& Colin, p. 335, pl. 5, figs. K-P.

Material. 22 specimens (samples A and C).

Illustrated specimens. LMA-00014, C, RV, f, Figure 4D; 1: $0.76 \mathrm{~mm}$, h: $0.43 \mathrm{~mm}$. LMA-00015, C, RV, m, Figure 4E; 1: $0.96 \mathrm{~mm}, \mathrm{~h}: 0.45 \mathrm{~mm}$.

Age. Santonian-Campanian.

Stratigraphic and geographic distribution. SantonianCampanian, Potiguar Basin (Viviers et al., 2000; Piovesan et al., 2014b; in this work).
Protocosta POT 1 Piovesan, Cabral \& Colin, 2014

(Figure 4F)

2014b Protocosta POT 1 Piovesan, Cabral \& Colin, p. 335, pl. 6, figs. Q-S.

Material. One specimen (sample C).

Illustrated specimen. LMA-00016, C, RV, Figure 4F; 1: $0.63 \mathrm{~mm}, \mathrm{~h}: 0.32 \mathrm{~mm}$.

Age. Santonian-Campanian.

Stratigraphic and geographic distribution. SantonianCampanian, Potiguar Basin (Piovesan et al., 2014b; in this work).

Trachyleberididae indet. gen. 6 Piovesan, Cabral \& Colin, 2014

(Figure 4G)

2014b Trachyleberididae indet. gen. 6. Piovesan et al., p. 348 , pl. 9 , figs. P-Q.

Material. Three specimens (samples A and C).

Illustrated specimen. LMA-00017, C, RV, Figure 4G; 1: $0.88 \mathrm{~mm}, \mathrm{~h}: 0.41 \mathrm{~mm}$

Age. Santonian-Campanian.

Stratigraphic and geographic distribution. SantonianCampanian, Potiguar Basin (Piovesan et al., 2014b; in this work).

Remarks. This is a very rare species registered by a single specimen in another locality of the Potiguar Basin by Piovesan et al. (2014b).

Subfamily BUNTONIINAE Apostolescu, 1961

Soudanella Apostolescu, 1961

Soudanella semicostellata (Grékoff, 1951)

(Figure 4H)

1951 Buntonia semicostellata Grékoff, p. 98, pl. 2, figs. 16-19. 1987 Buntonia (P.) semicostellata (Grékoff) - Okosun, p. 55-56, pl. 18, figs. 1-2; pl. 22, fig. 17.

1992 Protobuntonia semicostellata (Grékoff) - Okosun, p. 332, pl. 2, figs. 1-2.

2000 Protobuntonia sp. P6 Viviers, Koutsoukos, Silva-Telles \& Bengtson, p. 432, fig. 18, 7-8.

2014b Soudanella semicostellata (Grékoff, 1951) - Piovesan et al., p. 337, pl. 7, figs. O-Q.

Material. Five specimens (samples A and C).

Illustrated specimens. LMA-00018, C, RV, Figure 4H; 1: 1 $\mathrm{mm}, \mathrm{h}: 0.51 \mathrm{~mm}$.

Age. Santonian-Campanian.

Stratigraphic and geographic distribution. TuronianSantonian, Nigeria (Okosun, 1987, 1992); SantonianCampanian, Potiguar Basin (Viviers et al., 2000; Piovesan et al., 2014b; in this work); Campanian, Cameroon (Grékoff, 1951). 

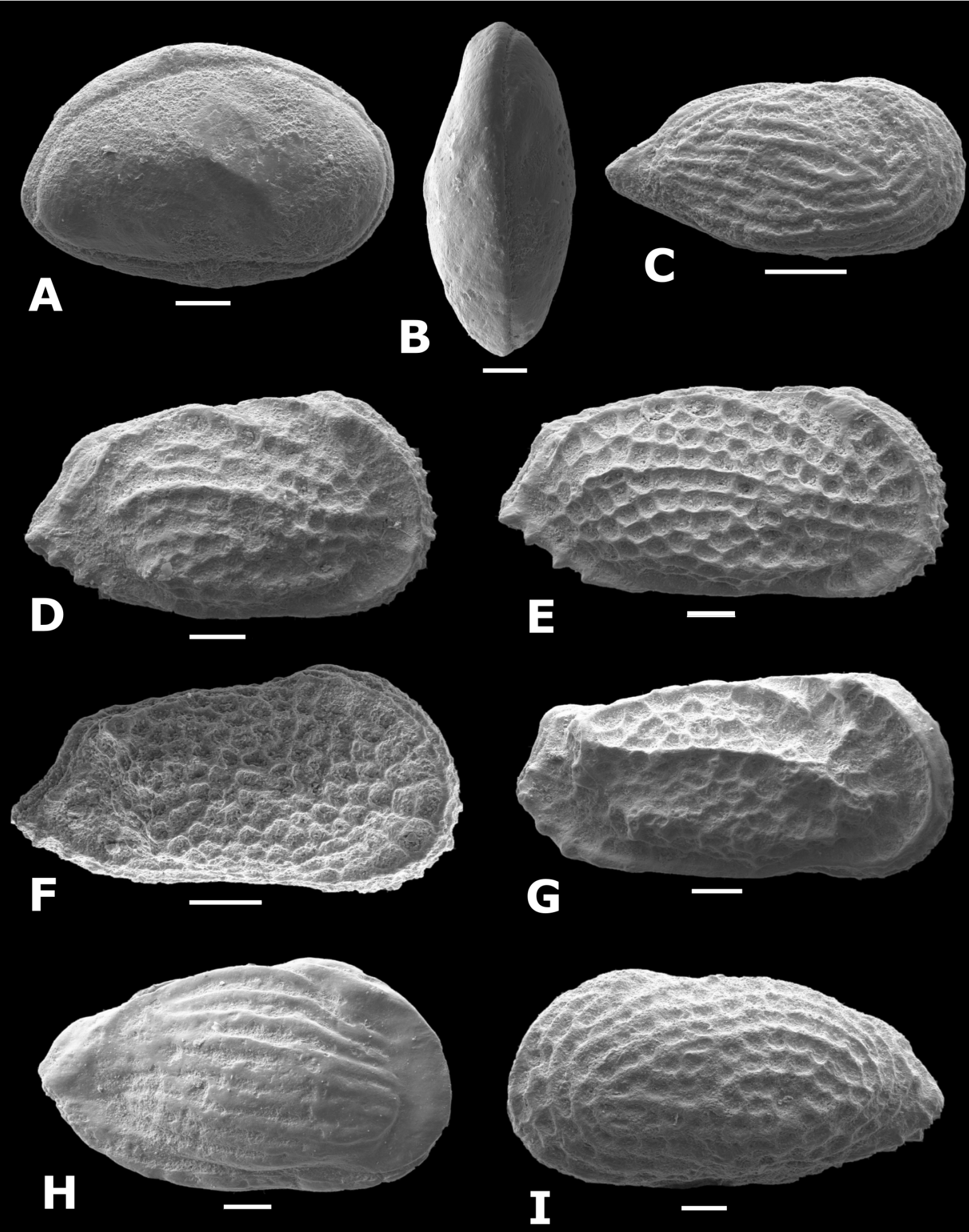

Figure 4. Ostracod species A, Ovocytheridea triangularis, C, RV (LMA-00011); B, Ovocytheridea triangularis, C, DV (LMA-00012); C, Perissocytheridea jandairensis, C, RV (LMA-00013); D, Protocosta babinoti, C, f, RV (LMA-00014); E, Protocosta babinoti, C, m, RV (LMA-00015); F, Protocosta POT 1, C, RV (LMA-00016); G, Trachyleberididae indet. gen. 6, C, RV (LMA-00017); H, Soudanella semicostellata, C, RV (LMA-00018); I, Leguminocythereis reymenti, C, LV (LMA-00019). Scale bars $=100 \mu \mathrm{m}$. 
Subfamily CAMPYLOCYTHERINAE Puri, 1960

Leguminocythereis Howe, 1936

Leguminocythereis reymenti Neufville, 1973

(Figure 4I)

1960 Leguminocythereis sp.- Reyment, p. 139, pl. 7, fig. 6. 1973 Leguminocythereis reymenti Neufville, p. 49-50. pl. 2, fig. 3, 4a-b.

1999 Leguminocythereis sp. Gebhardt, p. 89, figs. 6.5-6.9. 2000 Leguminocythereis reymenti Neufville - Delicio et al., p. 430 , figs. $17,1-2$.

2000 Leguminocythereis aff. L. reymenti - Viviers et al., p. 340-341, figs. 8.21-8.22.

2014b Leguminocythereis reymenti - Piovesan et al., p. 337, pl. 7, figs. R-T.

Material. Three specimens (sample C).

Illustrated specimens. LMA-00019, C, LV, Figure 4I; 1: 1 $\mathrm{mm}, \mathrm{h}: 0.5 \mathrm{~mm}$.

Age. Santonian-Campanian.

Stratigraphic and geographic distribution. TuronianSantonian, Nigeria (Reyment, 1960; Neufville, 1973; Gebhardt, 1999); Santonian-Campanian, Potiguar Basin (Delicio et al., 2000; Viviers et al., 2000; Piovesan et al., $2014 \mathrm{~b}$; in this work).

\section{DISCUSSION}

The Santonian-Campanian assemblages of the studied outcrop are composed of fifteen taxa, typical of marginal marine environments. Variations in taxonomic composition is attributed to both ecological and sedimentary processes, as previously discussed by Piovesan et al. (2015a). The sample A (base of the studied section) records abundance of the mixohaline species Ovocytheridea anterocompressa associated with Fossocytheridea potiguarensis and Perissocytheridea jandairensis, and scarce marine species. The same pattern was registered by Piovesan et al. (2015a) in this basin and referred to as the interval "Mixohaline/ Marine 2". In the Santonian-Campanian of the Santos Basin (southeastern Brazilian margin), the association Fossocytheridea-Perissocytheridea is considered an indicator of oligohaline/mesohaline environments (Bergue et al., 2011; Fauth et al., 2012), and a revision by Piovesan et al. (2015b) reinforced the euryhaline character of both genera. The marine influence in these assemblages is indicated by rare but typical marine ostracods (cytherellids and trachyleberidids), miliolids foraminifera, echinoid spines, bryozoan fragments and bivalve mollusks.

A remarkable decrease in diversity and abundance occurs in sample B. The abundance of Ovocytheridea anterocompressa and Perissocytheridea jandairensis decreases significantly concomitant with reduction in the incidence of marine mollusk fragments, mainly oysters, which is interpreted as decreased marine influence. The most drastic turnover, however, is registered in the sample $\mathrm{C}$, where diversified and typically marine assemblages dominated by cytherellids, bairdiids, and trachyleberidids replaced mixohaline taxa. Marine gastropods and echinoids are also recorded in this part of the section. The presence of Ovocytheridea triangularis and Cophinia ovalis in sample $\mathrm{C}$ demonstrate their ecological plasticity, occurring both in association with marine and brackish taxa, as also discussed by Piovesan et al. (2014b, 2015a). Sample D, the uppermost in the studied section, was barren of ostracods.

\section{CONCLUSIONS}

Ostracods are effective indicators of changes in the studied depositional environments in the Jandaíra Formation in terms of faunal turnover and abundance of some taxa. The extant diversity is incompletely known and deserves more study, because some taxa are still in open nomenclature, not only in this, but also in previous studies. This paper demonstrates that patterns observed by Piovesan et al. $(2014 a, b)$ are not restricted to the site of the core analyzed in those studies, but possibly characterizes broader areas of Jandaíra Formation.

\section{ACKNOWLEDGMENTS}

The authors wish to express their gratitude to N. Kumar Srivastava for support during field activities. We also thank the Laboratório de Dispositivos e Nanoestruturas (LDN-UFPE) and itt Fossil (UNISINOS) for the SEM pictures. J. Guzmán is thanked for the help with the study area figure.

\section{REFERENCES}

Andreu, B. 1996. Nouvelles espèces d'ostracodes du Turonien supérieur (?)-Coniacien (?)-Santonien de la région de Boulmane, Moyen Atlas, Maroc. Systématique, biostratigraphie, paléoécologie et paléobiogéographie des associations. In: S. Jardiné; I. de Klasz \& J.P. Debenay (eds.) Géologie de l'Afrique et de l'Atlantique Sud, Actes des Colloques de géologie d'Angers, p. 483-509.

Apostolescu, V. 1963. Essai de zonation par les Ostracodes dans le Crétacé du bassin du Sénégal. Revue de l'Institut Français du Pétrole, 18:1675-1694.

Araripe, P.T. \& Feijó, F.J. 1994. Bacia Potiguar. Boletim de Geociencias da Petrobras, 8:127-141.

Barros, C.L.; Piovesan, E.K. \& Agostinho, S.M.O. 2018. CretaceousPaleogene ostracods from the Paraíba Basin, northeastern Brazil. Journal of South American Earth Sciences, 83:117-136. doi:10.1016/j.jsames.2018.02.001

Bergue, C.T.; Fauth, G.; Vieira, C.E.L.; Santos, A.S. \& Viviers, M.C. 2011. New species of Fossocytheridea Swain \& Brown, 1964 (Ostracoda, Crustacea) in the Upper Cretaceous of Santos Basin, Brazil. Revista Brasileira de Paleontologia, 14:149-156. doi:10.4072/rbp.2011.2.03

Bismuth, H.; Boltenhagen C.; Donze, P.; Le Fevre, J. \& Saint-Marc, P. 1981. Le Crétacé moyen et supérieur du Djebel Semmama (Tunisie du Centre-Nord). Bulletin des Centres de Recherches Exploration Production elf Aquitaine, 5:193-267. 
Delicio, M.P.; Coimbra, J.C. \& Carreño, A.L. 2000. Cretaceous marine Ostracoda from the Potiguar Basin, northeastern Brazil. Neues Jahrbuch für Geologie und Palaöntologie, Abhandlungen, 215:321-345. doi:10.1127/njgpa/215/2000/321

Fauth, G.; Colin J.-P.; Koutsoukos, E.A. \& Bengtson, P. 2005. Cretaceous/Tertiary boundary ostracodes from the Poty Quarry, Pernambuco, Northeastern Brazil. Journal of South American Earth Sciences, 19:285-305. doi:10.1016/j.jsames.2005.01.007

Fauth, G.; Santos, A.S.; Vieira, C.E.L.; Bergue, C.T.; Musachio, E.A.; Ferreira, E.P.; Escamilla, J.H.; Carvalho, M.A.; Viviers, M.C. \& Fauth, S.B. 2012. Bioestratigrafia integrada do Cretáceo Superior da Bacia de Santos: ostracodes, carófitas e palinomorfos. Boletim de Geociências da Petrobras, 20:229-258.

Fauth, G.; Santos Filho, M.A.B; Ceolin, D. \& Gradstein, F. 2018. Cretaceous marine ostracod biostratigraphy of the South Atlantic Ocean: an overview. Revue de Micropaléontologie, 61:139-153. doi:10.1016/j.revmic.2018.10.003

Gebhardt, H. 1999. Cenomanian to Coniacian ostracodes from the Nkalagu area (SE Nigeria): biostratigraphy and palaeoecology. Paläontologische Zeitschrift, 73:77-98. doi:10.1007/BF02987984

Grékoff, N. 1951. Quelques ostracodes nouveaux du Sénonien supérieur du Cameroun. Revue de l'Institut Français du Petrole et Annales des Combustibles liquides, 6:53-59.

Horne, D.J. 2005. Ostracoda. In: R.C. Selley; R.M. Cocks \& I.R. Plimer (eds.) Encyclopedia of Geology, Elsevier, p. 453-463. doi:10.1016/B0-12-369396-9/00511-6

Mohriak, W.U. 2003. Bacias sedimentares da margem continental brasileira. In: L.A. Bizzi; C. Schobbenhaus; R.M. Vidotti \& J.H. Gonçalves (eds.) Geologia, tectônica e recursos minerais do Brasil, CPRM, p. 87-94.

Monteiro, M.C. \& Faria, T.R. 1988. Planície de maré no poço 9-MO-13-RN, Formação Jandaíra - um exemplo do passado. In: CONGRESSO BRASILEIRO DE GEOLOGIA, 35, 1998. Anais, Belém, p. 809-823.

Neufville, E.M.H. 1973. Upper Cretaceous-Palaeogene Ostracoda from the South Atlantic. Uppsala, Palaeontological Institution, University of Uppsala, 205 p. (Publications 1).

Okosun, E.A. 1987. Ostracod biostratigraphy of the Eastern Dahomey Basin, Niger Delta and the Benue Trough of Nigeria. Geological Survey of Nigeria, $151 \mathrm{p}$.

Okosun, E.A. 1992. Cretaceous ostracod biostratigraphy from Chad Basin in Nigeria. Journal of African Earth Sciences, 14:327-339. doi:10.1016/0899-5362(92)90036-C

Pessoa Neto, O.C.; Soares, U.M.; Silva, J.G.F.; Roesner, E.H.; Florencio, C.P. \& Souza, C.A.V. 2007. Bacia Potiguar. Boletim de Geociências da Petrobras, 15:357-387.
Piovesan, E.K.; Bergue, C.T.; Fauth, G.; Viviers, M.C. $2015 \mathrm{a}$. Palaeoecology of ostracods from the Late Cretaceous from northeastern Brazil and its relation to sequence stratigraphy. Palaeogeography, Palaeoclimatology, Palaeoecology, 424:4048. doi:10.1016/j.palaeo.2015.02.015

Piovesan, E.K.; Cabral, M.C.; Boavida, E.A.; Colin, J.P. \& Fauth, G. 2015b. Fossocytheridea Swain \& Brown and Perissocytheridea Stephenson (Ostracoda): insights into paleosalinity gradients of Late Cretaceous deposits from Brazil and Portugal. Revista Brasileira de Paleontologia, 18:21-30. doi:10.4072/ rbp.2015.1.02

Piovesan, E.K.; Cabral, M.C.; Colin, J.-P.; Fauth, G. \& Bergue, C.T. 2014a. Ostracodes from the Upper Cretaceous deposits of the Potiguar Basin, Northeastern Brazil: taxonomy, paleoecology and paleobiogeography, part 1: Turonian. Carnets de Géologie, 14:211-252. doi:10.4267/2042/54003

Piovesan, E.K.; Cabral, M.C.; Colin, J.-P.; Fauth, G. \& Bergue, C.T. 2014b. Ostracodes from the Upper Cretaceous deposits of the Potiguar Basin, Northeastern Brazil: taxonomy, paleoecology and paleobiogeography, part 2: Santonian-Campanian. Carnets de Géologie, 14:315-351. doi:10.4267/2042/54151

Reyment, R.A. 1960. Studies on Nigerian Upper Cretaceous and lower Tertiary Ostracoda: part 1. Senonian and Maastrichtian Ostracoda. Acta Universitatis Stockholmiensis, Stockholm Contributions in Geology, 7:1-239.

Santos Filho, M.A.B.; Piovesan, E.K.; Fauth, G. \& Srivastava, N.K. 2015. Paleoenvironmental interpretation through the analysis of ostracodes and carbonate microfacies: study of the Jandaíra Formation, Upper Cretaceous, Potiguar Basin. Brazilian Journal of Geology, 45:23-34. doi:10.1590/23174889201500010002

Vivière, J.L. 1985. Les Ostracodes du Crétacé Supérieur (Vraconien à Campanien basal) de la région de Tébessa (Algérie du NordEst): stratigraphie, paléoécologie, systématique. Université Pierre et Marie Curie, Ph.D. thesis, 261 p.

Viviers, M.C. 1995. Ostracodes marinhos na Bacia Potiguar: aplicação nos estudos bioestratigráficos do Cretáceo. Anais da Academia Brasileira de Ciências, 67:392-393.

Viviers, M.C.; Koutsoukos, E.A.M.; Silva-Telles, A.C. \& Bengtson, P. 2000. Stratigraphy and biogeographic affinities of the late Aptian-Campanian ostracods of the Potiguar and Sergipe basins in northeastern Brazil. Cretaceous Research, 21:407-455. doi:10.1006/cres.2000.0205

Received in 07 August, 2019; accepted in 30 March, 2020. 